\title{
Contribución de las actividades de extensión a la formación en Terapia Ocupacional
}

\author{
Contribution of the extension activities to \\ Occupational Therapy formation
}

Contribuição das atividades de extensão para a formação em Terapia Ocupacional

\author{
Mirela de Oliveira Figueiredo ${ }^{7}$ \\ Izamara Pereira Paterra ${ }^{2}$ \\ Carla Regina Silva ${ }^{3}$ \\ Isadora Cardinalli ${ }^{4}$
}

Recibido: 30 de mayo 2019 • Enviado para modificación: 19 de febrero 2020 • Aceptado: 15 de junio 2020

Figueiredo, M.O., Paterra, I.P., Silva, C.R. y Cardinalli, I. (2020). Contribución de las actividades de extensión a la

formación en Terapia Ocupacional. Revista Ocupación Humana, 20 (1), 7-26. https://doi.org/10.25214/25907816.946

\section{RESUMEN}

La extensión universitaria constituye una actividad académica indisociable de la enseñanza y de la investigación. Considerando su importancia, este artículo presenta una investigación cuyo objetivo fue analizar y describir las actividades de extensión realizadas en la carrera de Terapia Ocupacional de la Universidad Federal de San Carlos (São Paulo, Brasil) entre los años 2000 y 2017. Se realizó un estudio descriptivo de tipo exploratorio con enfoque cuantitativo. Los datos se recolectaron en el acervo digital ProExWeb, sistema de gestión de las actividades de extensión, utilizando el término terapia ocupacional y los nombres de docentes en el periodo estudiado. Se encontraron 425 actividades vinculadas a 39 coordinadores y con la participación de 1.380 estudiantes. 354 actividades fueron realizadas en o con comunidades, que alcanzaron a 73.264 personas de diferentes cursos de vida, en distintas áreas y campos. Las actividades abar-

\footnotetext{
${ }^{1}$ Terapeuta ocupacional. Magíster en Salud del Niño y del Adolescente. Doctora en Educación Especial. Profesora, Universidad Federal de São Carlos. San Carlos, Brasil. mirelafigueiredo@gmail.com. (iD) https://orcid.org/0000-0003-0101-0115

${ }^{2}$ Terapeuta ocupacional. Residente, Universidad de São Paulo. San Pablo, Brasil. izamarapereirapaterra@gmail.com. (iD) https://orcid.org/0000-0001-5320-2755

${ }^{3}$ Terapeuta ocupacional. Magíster en Educación. Doctora en Educación. Profesora, Universidad Federal de São Carlos. San Carlos, Brasil. carlars@ufscar.br. (iD) http://orcid.org/0000-0002-7079-8340

${ }^{4}$ Terapeuta ocupacional. Magíster y estudiante de Doctorado en Terapia Ocupacional. Universidad Federal de São Carlos. San Carlos, Brasil. isadora.cardinalli@gmail.com. iD https://orcid.org/0000-0001$\underline{8918-3162}$
} 
can diversas propuestas, objetivos, públicos, con integración con otros departamentos, servicios y sectores públicos. Así, se cumple con la principal meta de la extensión universitaria, que es la relación con la comunidad y responder a sus demandas, articulándose con la enseñanza y con la investigación para favorecer la formación discente implicada, ética y responsable.

\title{
PALABRAS CLAVE
}

extensión universitaria, cambio social, participación de la comunidad, servicio universitario

\begin{abstract}
University extension is an academic activity that cannot be separated from teaching and research. Considering its importance, this article presents an investigation aiming to analyze and describe the extension activities carried out by the Occupational Therapy course at the Federal University of São Carlos (São Paulo, Brazil) from 2000 to 2017. A descriptive exploratory study was carried out, with a quantitative approach. Data were collected in the ProExWeb digital archive, a management system for the university's extension activities, using the term "occupational therapy" and the names of lecturers in the period studied. Among the findings, 425 activities were linked to 39 coordinators, and with the participation of 1,380 students. 354 activities were carried out in or with the communities, reaching 73,264 people from different walks of life, in different areas and fields. The activities cover several proposals, objectives, audiences and integrate different departments, services, and the public sector. Thus, university extension's primary objective -its relationship with the community and the response to their demands- is fulfilled by assembling it with teaching and research to promote an engaged, ethical and responsible student formation.
\end{abstract}

\section{KEY WORDS}

university extension, social change, community participation, university service

\section{RESUMO}

A extensão universitária constitui uma atividade acadêmica inseparável do ensino e da pesquisa. Por considerar sua importância, este artigo apresenta uma investigação com o objetivo de analisar e descrever as atividades de extensão realizadas pelo curso de Terapia Ocupacional da Universidade Federal de São Carlos (São Paulo, Brasil), entre 2000 e 2017. Foi realizado um estudo do tipo exploratório descritivo, com abordagem quantitativa. Os dados foram coletados no acervo digital ProExWeb, um sistema de gerenciamento de atividades de extensão da universidade. Utilizou-se o termo "terapia ocupacional" e os nomes dos professores no período estudado. Foram encontradas 425 atividades relacionadas a 39 coordenadores e com a participação de 1.380 alunos. Realizaram-se 354 atividades nas ou com as comunidades, atingindo 73.264 pessoas de diferentes estilos de vida, em diferentes áreas e campos. As atividades abrangem várias propostas, objetivos, públicos, bem como integração com outros departamentos, serviços e setores públicos. Assim, foi cumprido o principal objetivo da extensão universitária, que é o relacionamento com a comunidade e a resposta às demandas - articuladas com o ensino e a pesquisa, o que promove uma formação discente engajada, ética e responsável.

\section{PALAVRAS-CHAVE}

extensão universitária, mudança social, participação da comunidade, serviço universitário 


\section{Introducción}

La extensión universitaria tiene un papel estratégico en la articulación de la enseñanza con la investigación y en la preservación y difusión de la cultura. De esta forma, favorece la formación de individuos reflexivos y comprometidos con una sociedad menos injusta (Fresan, 2004) y posibilita el intercambio de conocimiento entre la universidad y la sociedad (Ortiz y Morales, 2011).

Hay distintas concepciones, prácticas y gestiones para lograr la extensión universitaria en Latinoamérica. En la región, la extensión estuvo presente desde 1918, a partir de la Reforma Universitaria impulsada por la Universidad Nacional de Córdoba, en Argentina, y generó repercusiones importantes en el escenario educativo. Estudiantes de clase media presionaban para lograr el acceso a la universidad, reivindicaban el cambio en la educación y en la administración, y estaban en contra de la interferencia y el control del clero y la oligarquía. De esta manera, la Reforma incluyó una serie de planteamientos políticos y sociales, entre los cuales aparece el fortalecimiento de la función social de la universidad, además de la proyección de la cultura universitaria y la preocupación por los problemas nacionales (Tünnermann, 2000; Farias, 2015).

En este contexto, algunas universidades latinoamericanas emprendieron reformas. Entre ellas se destaca el surgimiento de la misión social de los universitarios, que consistía en manifestar la obligación ineludible de compartir la cultura y los conocimientos con el "desposeído o ignorante", promovien- do la justicia social. Así fue conferida a la extensión la responsabilidad de promover y coordinar las acciones que, para ese fin, implementaban las universidades (Serna, 2007).

Otro marco histórico importante fue la Declaración de la Unesco resultante de la Conferencia Mundial sobre Educación Superior de 1998, donde se afirmó que esta debía reforzar sus funciones de servicio a la sociedad y que sus acciones debían estar relacionadas con "la erradicación de la pobreza, el hambre, el analfabetismo, la violencia, la intolerancia, el deterioro del medio ambiente, mediante esfuerzos interdisciplinarios para analizar los diferentes problemas" (Ortiz y Morales, 2011, p.352).

Desde la década de 1980, el modelo económico neoliberal presente en Latinoamérica penetró todas las instancias de las políticas públicas y sociales, incluyendo las educativas y de enseñanza superior (Harvey, 2014). Sin embargo, las universidades han vivido procesos de cambio, adaptación y resistencia frente a las implicaciones de este modelo, dentro de los cuales la extensión universitaria se ha presentado como una respuesta conveniente y efectiva.

Las universidades públicas, en especial las latinoamericanas, tienen actualmente un desafío extremadamente importante, la búsqueda de su autonomía y democratización; a la vez que están sometidas a las políticas propuestas por el sistema neoliberal, que define su calidad, eficacia y eficiencia a partir de modelos empresariales y del mercado que provocan un giro en su función social. 
La extensión universitaria -o vinculación con el medio, como algunas veces es conocida- sufre las contradicciones intrínsecas a este contexto, pues posee las características para regular y promover la mercantilización de la universidad. A su vez, posibilita intercambios y amplía las posibilidades de formación ética, política y consciente en el contacto con grupos, comunidades y movimientos sociales dirigido a la construcción de conocimiento dialógico y liberador (Freire, 1987), y en la búsqueda constante de la transformación social, desde la función social de la universidad.

\section{Las actividades de extensión en la universidad pública brasileña}

Actualmente, la extensión en la universidad pública brasileña constituye la práctica académica indisociable de las actividades de enseñanza e investigación; de la misma forma, está articulada con las demandas sociales, según su naturaleza intrínseca (Pedrazzi y Yamamoto, 2013).

Históricamente la extensión fue, de las tres acciones universitarias en Brasil, la última en ser creada; su implementación se renovó a través del tiempo, con las distintas interpretaciones y la autonomía de cada institución (Paula, 2013). Con frecuencia la extensión universitaria se ha mantenido al margen, en un segundo plano respecto a la investigación y la enseñanza (Llorens, 1992).

La propia creación de las universidades es tardía en Brasil, con acceso limitado y exclusivo para las élites. Los registros de acciones de extensión datan de 1911, realizadas bajo la in- fluencia de vertientes y conceptos tanto europeos como norteamericanos (Nogueira, 2001). La experiencia latinoamericana también influenció este escenario y la extensión universitaria fue comprendida en relación con los movimientos sociales, desde el marco histórico del Movimiento de la Reforma de Córdoba (1918).

En el Estatuto de las Universidades Brasileñas (1931), la extensión es citada como aquella capaz de elevar el nivel de la cultura del pueblo. Entre tanto, las actividades ofrecidas consistían en una suerte de "especialización" para exalumnos, beneficiando a aquellos que tenían poder político y socioeconómico (Nogueira, 2001). Así, una determinada cultura fue valorada y diseminada, y la extensión se realizó de forma vertical. Una "cosificación" del otro, como cuando uno de los actores impone sus creencias, acciones y opiniones para "domesticarlo", hecho al parecer inocente o bienintencionado (Freire, 1985).

Fleuri (1989) refiere que en Brasil, hasta 1960, las experiencias surgidas en el ámbito de la extensión universitaria estaban relacionadas, esencialmente, con los intereses e ideologías de la clase dominante, ofreciendo servicios, información y actividades artísticas para satisfacer la curiosidad de las élites. En 1960, colectivos de estudiantes realizaron acciones de extensión sin vínculo con las universidades. La Unión Nacional de los Estudiantes realizó seminarios en 1960 y 1961, con la temática de la Reforma Universitaria y en defensa de la creación de universidades con acciones de extensión, dentro de las cuales la reflexión sobre la práctica y el compromiso con las clases populares eran ejes centrales del nuevo sistema de enseñanza (Nogueira, 2001). 
Sin embargo, durante la Dictadura Militar (1964 - 1985) hubo una extinción de los órganos de representatividad, como la Unión Nacional de Estudiantes, y el Gobierno propuso la extensión de forma asistencialista para legitimar la dictadura y mantener a los estudiantes ocupados (Minto, 2011). La Ley Básica de Reforma Universitaria (1968) afirmaba la extensión universitaria como una estrategia para extender el conocimiento producido por la universidad a través de cursos y de la prestación de servicios de forma asistencialista (Nogueira, 2001).

Durante la década de los setenta, se reafirmó la extensión como el medio a través del cual la universidad, por un lado, atiende a otras instituciones y a la población y, por otro, se realimenta con la enseñanza y la investigación (Fleuri, 1989). Los cambios empezaron en los años ochenta, cuando el proceso de redemocratización y las luchas de los movimientos sociales fueron más fuertes, alegando que la formulación de políticas públicas debería garantizar la defensa de los derechos sociales, englobando a poblaciones históricamente excluidas, tales como niños, adolescentes, ancianos y grupos que sufrían discriminación étnica, racial y de género (Paula, 2013), entre otros.

La Constitución Federal de 1988 dispone que las universidades gozan de autonomía didáctico-científica, administrativa y de gestión financiera y patrimonial, y deben obedecer al principio de indisociabilidad entre enseñanza, investigación y extensión (Presidência da República, 1988).

Desde el 2003, los proyectos de extensión han sido apoyados y fomentados por varios ministerios, así como por el
Consejo Nacional de Desarrollo Científico y Tecnológico, que ofrece ediciones específicas con la temática de la extensión universitaria (Fórum de Pró-Reitores de Extensão das Universidades Públicas Brasileiras -Forproex, 2006). No obstante, la inestabilidad política y económica actual del país genera gran inseguridad y cortes en la financiación pública, al tiempo que expande las gestiones pautadas en los modelos neoliberales, que buscan apoyo en el sector privado para realizar innumerables actividades y encuentran en la extensión universitaria la puerta de entrada.

Sousa (2007) presenta dos niveles de mercantilización de la universidad pública en las últimas décadas. El primero consiste en inducir a la universidad a sobreponerse a la crisis financiera mediante la generación de ingresos propios, especialmente a través de alianzas con el capital, sobre todo industrial. El segundo nivel intenta eliminar la distinción entre universidad pública y privada, transformándola en una empresa, una entidad que produce como y para el mercado.

La crítica no está anclada en que esta forma no debiera existir en la universidad pública, pero es inaceptable creer que todas las actividades, en todos los ámbitos de la extensión universitaria, áreas y campos profesionales deban responder a esta misma lógica, sin considerar los problemas, demandas, exclusiones y desigualdades sociales generadas por ese sistema. Sobre todo, hay que rescatar y fortalecer la universidad como una herramienta importante para la transformación social.

La formación técnica en Terapia Ocupacional, desde su aparición en Brasil en 1950, requiere conocimientos 
teóricos y prácticos e involucra a docentes, estudiantes, sujetos y comunidades, quienes son considerados prioritarios en la práctica. Así, la extensión se presenta como estrategia eficaz, coherente e integrada a las actividades de enseñanza e investigación.

En la década de 1960, las carreras se tornaron oficialmente de nivel superior, siendo ofrecidas por universidades. En los años setenta la profesión pasó por su primer momento de crecimiento, tanto de los programas de formación como de las vacantes universitarias públicas (Soares, 1991). Durante la Dictadura Militar hubo una fuerte interferencia en el perfil de la enseñanza superior pública y en la movilización de los sectores sociales (Fausto, 2009) con los cuales se involucraba la Terapia Ocupacional.

Al final del período de represión, diferentes movimientos por la redemocratización y por los derechos de las poblaciones marginadas contribuyeron a la defensa del papel técnico, ético y político de las y los terapeutas ocupacionales. Estos efectos se reflejaron directamente en la relación con la práctica profesional y en la formación universitaria desde los años ochenta (Galheigo, 2016).

De acuerdo con Apablaza (2015), los complejos procesos de cambio de concepciones y prácticas, que afectan a la Terapia Ocupacional, interfieren y exigen la adecuación constante de los programas, allí las experiencias prácticas son valoradas y consideradas componentes claves de la formación profesional.

Cardinalli (2017) afirma que, en las últimas décadas, las y los terapeutas ocupacionales brasileños se han dedicado a estudiar, producir e implementar referencias teórico-metodológicas, entendiendo la necesidad de producir y nombrar términos, conceptos, razonamientos, perspectivas y modelos teórico-prácticos que expliquen la diversidad e, incluso, la transdisciplinariedad de las prácticas. En ese sentido, las producciones teóricas y prácticas deben ofrecer soporte y ampliar las reflexiones y las posibilidades de formación y actuación profesional. Por lo tanto, las experiencias académicas deberían garantizar la diversidad de prácticas; allí la extensión universitaria es una herramienta clave.

El curso de Terapia Ocupacional de la Universidad Federal de São Carlos (UFSCar) fue fundado en 1978. Actualmente, su programa curricular ha sido reformulado y cuenta con una metodología que combina práctica y teoría. El campo de aprendizaje construido a partir de la práctica de extensión abarca no solo a docentes y discentes, sino a todas las personas involucradas (Coordenação do curso de bacharelado em Terapia Ocupacional, 2011; Junior, 2013; Silva et al., 2013). En esta universidad se comprende a la extensión como: publicaciones y difusión del conocimiento; eventos culturales, científicos, artísticos, deportivos y otros; producción de conocimiento con el objetivo de incrementar y mejorar la atención directa o indirecta a la sociedad; prestación de servicios, asesorías o consultorías; actividades de divulgación, difusión y transferencia de tecnologías; cursos de especialización, perfeccionamiento, actualización científica, cultural y artística; entre otros 
(Conselho de Extensão da Universidade Federal de São Carlos, 2016).

Esta investigación tuvo como objetivo describir las actividades de extensión realizadas por docentes del Departamento de Terapia Ocupacional de la Universidad Federal de São Carlos (São Paulo, Brasil) y analizar su relación con la formación frente el compromiso social de la universidad pública.

\section{Metodología}

Se realizó un estudio exploratorio descriptivo, con enfoque cuantitativo para el tratamiento de los datos, utilizando análisis estadístico simple (frecuencias y medias) (Goodwin, 1995).

Los datos se recolectaron a través del acervo digital de la Pro-Rectoría de Extensión Universitaria UFSCar, en ProExWeb, un sistema para la gestión en línea de la extensión de la Universidad en el que se realiza la inserción continua de estas actividades, así como de los procedimientos y pruebas internas de las propuestas.

Para la búsqueda, realizada en abril de 2018, se utilizó la palabra clave terapia ocupacional y los nombres de las y los docentes activos, jubilados o transferidos del Departamento de Terapia Ocupacional entre los años 2000 y 2017 . Este período está justificado porque el sistema de registro de actividades existe desde la década de 2000. Se llevaron a cabo cuatro procedimientos de colecta de datos:

- Búsqueda con la palabra clave terapia ocupacional en el campo título y el acrónimo DTO (Departamento de Terapia Ocupacional) en el campo sector.
- Búsqueda con la palabra clave terapia ocupacional en el campo palabras clave y uso del acrónimo DTO en el campo sector.

- Búsqueda con la palabra clave terapia ocupacional en el campo resumen y el acrónimo DTO en el campo sector.

-Búsqueda con la inserción del nombre de cada profesor(a) y exprofesor(a) del Departamento de Terapia Ocupacional, incluyendo aquellas personas jubiladas o transferidas del Departamento en el periodo establecido, en el campo coordinación.

Se elaboró un formulario para registrar los datos, que contempló las siguientes categorías de actividad: coordinador, título, programa, año de realización, público, lugar y miembros del equipo. Las actividades se ingresaron en una hoja de cálculo, eliminando repeticiones. Todos los datos fueron tabulados en hojas de cálculo del programa Excel®. Se realizaron cruces, análisis, gráficos y tablas para la presentación de los resultados.

\section{Resultados}

\section{Actividades de extensión a lo largo de los años}

Fueron encontradas 409 actividades de extensión vinculadas a docentes del Departamento de Terapia Ocupacional de la Universidad Federal de São Carlos entre 2000 y 2017 (figura 1).

La mayoría de las actividades de extensión tuvieron una duración de al menos ocho meses, con acciones semanales en los campos de práctica, o sea, no se 
trató de actividades puntuales. Además, del total, 70 actividades (16,47\%) tuvieron, por lo menos, una segunda oferta. Esos datos demuestran la permanencia y continuidad de las acciones propuestas, por lo que es posible pensar que son pertinentes y se vinculan con las personas y las comunidades.

La figura 1 muestra una diferencia entre las décadas estudiadas, entre 2000 y 2009 hay una media de 12,8 activida- des de extensión, mientras en la década posterior ese número aumenta a 31,9. Esto responde al aumento de docentes coordinadores, es decir, aumentan las propuestas porque hay más profesoras y profesores en el Departamento. Tal incremento es reflejo de las políticas educativas de ese período, que ampliaron el número de docentes en las universidades públicas y ofrecieron mayores posibilidades de inversión. No obstante, desde 2016 hay una baja en esos números, lo

Figura 1. Distribución de actividades de extensión por años y docentes a cargo.

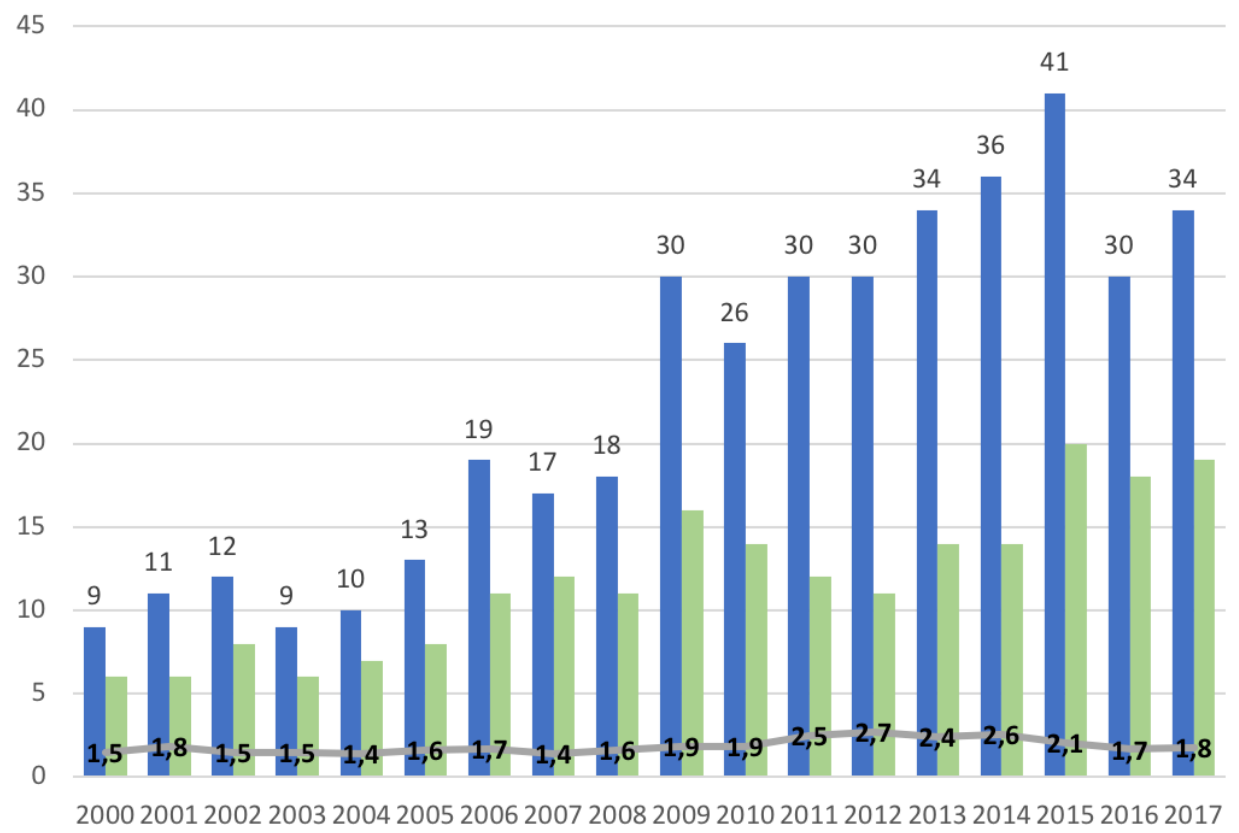

Fuente: elaboración propia. 
que coincide con los cambios de gestión política ocurridos en Brasil, los cuales significaron transformaciones estructurales, culturales e ideológicas, así como el retiro de inversiones.

Se verificó que 19 profesoras realizaron 322 actividades, una media de 16 proyectos por coordinación. Además, la totalidad de docentes realizó actividades de extensión universitaria, unos con mayor frecuencia que otros y algunos no durante todos los años analizados. Aun así, la extensión se revela como actividad intrínseca del docente de la carrera de Terapia Ocupacional de esta universidad pública.

\section{Actividades incluidas en programas de extensión}

Las actividades están vinculadas a programas de extensión, que a menudo se vinculan con los seis laboratorios de enseñanza, investigación y extensión existentes en el Departamento de Terapia Ocupacional (tabla 1).

Se resalta que los programas fueron creados en periodos distintos y poseen un número diferenciado de docentes vinculados, lo que explica las variaciones en la cantidad de actividades y los años en que se ofrecieron.

Otros cuatro programas están actualmente inactivos (Atención a la Disfunción Física y la Salud, Actuación de
Terapia Ocupacional en Gerontología, Promoviendo y Recuperando a Salud del Trabajador en la Empresa, Educación Continua en Letras), por eso no se incluyen en la tabla 1. Sin embargo, entre 1999 y 2012 estuvieron activos en el campo de la gerontología, las disfunciones físicas, los derechos humanos de niños, niñas y adolescentes, y la atención e investigación en salud, desarrollando 23 proyectos.

Además, se identificaron 24 actividades coordinadas por docentes del Departamento de Terapia Ocupacional entre 2003 y 2017, vinculadas a ocho programas pertenecientes a otros seis departamentos de la Universidad en las áreas de educación musical, formación continuada, cooperativas populares, servicios de salud, atención e investigación en salud, salud del trabajador y salud mental en el contexto de la reforma psiquiátrica.

\section{Caracterización de las actividades desarrolladas}

En cuanto a los tipos de actividades realizadas, se verificó que 354 fueron proyectos dirigidos a la comunidad, 52 eventos académicos (semanas de estudios, seminarios, simposios, cursos y otros), siete actividades curriculares de integración enseñanza, investigación y extensión ${ }^{5}$, y cinco destinadas a la publicación de los Cuadernos Brasileños de Terapia Ocupacional' ${ }^{6}$.

\footnotetext{
${ }^{5}$ Estas son actividades curriculares complementarias insertadas en los currículos de pregrado. Tienen una duración semestral de 60 horas; los estudiantes pueden escoger cuál quieren cursar. Esta modalidad de actividad de extensión regula la integración entre enseñanza, investigación y extensión y oficializa créditos curriculares a estudiantes y coordinadores.
}

${ }^{6}$ Considerando su denominación anterior: Cuadernos de Terapia Ocupacional de la UFSCar. 
Tabla 1. Programas con actividades de extensión activas.

\begin{tabular}{|c|c|c|}
\hline Programa & Público & $\mathrm{N}^{\circ}$ de actividades (año) \\
\hline $\begin{array}{l}\text { Actividad Humana, } \\
\text { Producción Artístico- } \\
\text { cultural y Terapia } \\
\text { Ocupacional }\end{array}$ & $\begin{array}{l}\text { Niños, jóvenes, adultos y adultos } \\
\text { mayores. Estudiantes y profesionales, } \\
\text { especialmente terapeutas } \\
\text { ocupacionales. }\end{array}$ & $\begin{array}{l}4(2013), 6 \text { (2014), } 6 \\
(2015), 5 \text { (2016), } 8 \text { (2017). }\end{array}$ \\
\hline $\begin{array}{l}\text { Laboratorio de } \\
\text { Actividades y } \\
\text { Desarrollo }\end{array}$ & $\begin{array}{l}\text { Niños y adolescentes con trastornos } \\
\text { del desarrollo, familias, profesionales } \\
\text { de salud y educación. }\end{array}$ & $\begin{array}{l}2 \text { (2000), } 1(2001), 2 \\
(2002), 3(2003), 4(2004) \\
4(2005), 6(2006), 4 \\
(2007), 5(2008), 7(2009) \\
6(2010), 9(2011), 8 \\
(2012), 10(2013), 13 \\
(2014), 2(2015), 5(2016) \\
6(2017) .\end{array}$ \\
\hline $\begin{array}{l}\text { METUIA - Terapia } \\
\text { Ocupacional en el } \\
\text { Campo Social }\end{array}$ & $\begin{array}{l}\text { Niños, adolescentes y adultos en } \\
\text { proceso de ruptura de las redes de } \\
\text { soporte social; estudiantes y } \\
\text { profesionales de la salud, educación, } \\
\text { cultura, especialmente de Terapia } \\
\text { Ocupacional. }\end{array}$ & $\begin{array}{l}2 \text { (2000), } 1 \text { (2001), } 2 \\
(2002), 2 \text { (2003), } 3(2004) \\
5(2005), 5(2006), 2 \\
(2007), 2(2008), 6(2009) \\
4(2010), 3(2011), 4 \\
(2012), 4(2013), 6(2014), \\
8(2015), 6(2016), 5 \\
(2017) .\end{array}$ \\
\hline $\begin{array}{l}\text { Programa de Acción } \\
\text { e Investigación en } \\
\text { Terapia Ocupacional } \\
\text { en Disfunción Física } \\
\text { del Adulto }\end{array}$ & $\begin{array}{l}\text { Estudiantes; usuarios de la Unidad de } \\
\text { Salud Universitaria; profesionales; } \\
\text { personas con disturbios o disfunciones } \\
\text { sensoriales o motoras, o en riesgo de } \\
\text { malestares ocupacionales; } \\
\text { profesionales de salud, especialmente } \\
\text { terapeutas ocupacionales; empresas e } \\
\text { instituciones de salud y educación. }\end{array}$ & $\begin{array}{l}6 \quad(2009), 4 \quad(2013), 5 \\
(2010), 1(2014), 6(2011), \\
3 \quad(2015), 5 \quad(2012), 6 \\
(2016), 3(2017) .\end{array}$ \\
\hline Calidad de Vida & $\begin{array}{l}\text { Funcionarios, docentes y sus } \\
\text { familiares. }\end{array}$ & $1(2007)$ \\
\hline $\begin{array}{l}\text { Terapia Ocupacional } \\
\text { en Psiquiatría y Salud } \\
\text { Mental }\end{array}$ & $\begin{array}{l}\text { Usuarios de los servicios de psiquiatría } \\
\text { y sus familiares, terapeutas } \\
\text { ocupacionales, estudiantes de pregrado } \\
\text { y posgrado. }\end{array}$ & $\begin{array}{l}1(2000), 1(2001), 1 \\
(2002), 2 \text { (2005), } 2 \text { (2007), } \\
2(2008), 1(2009), 4 \\
(2010), 7(2011), 7(2012), \\
8(2013), 5(2014), 6 \\
(2015), 7(2016), 9 \text { (2017). }\end{array}$ \\
\hline
\end{tabular}

Fuente: elaboración propia. 
Figura 2. Distribución de actividades según campos o áreas de prácticas.

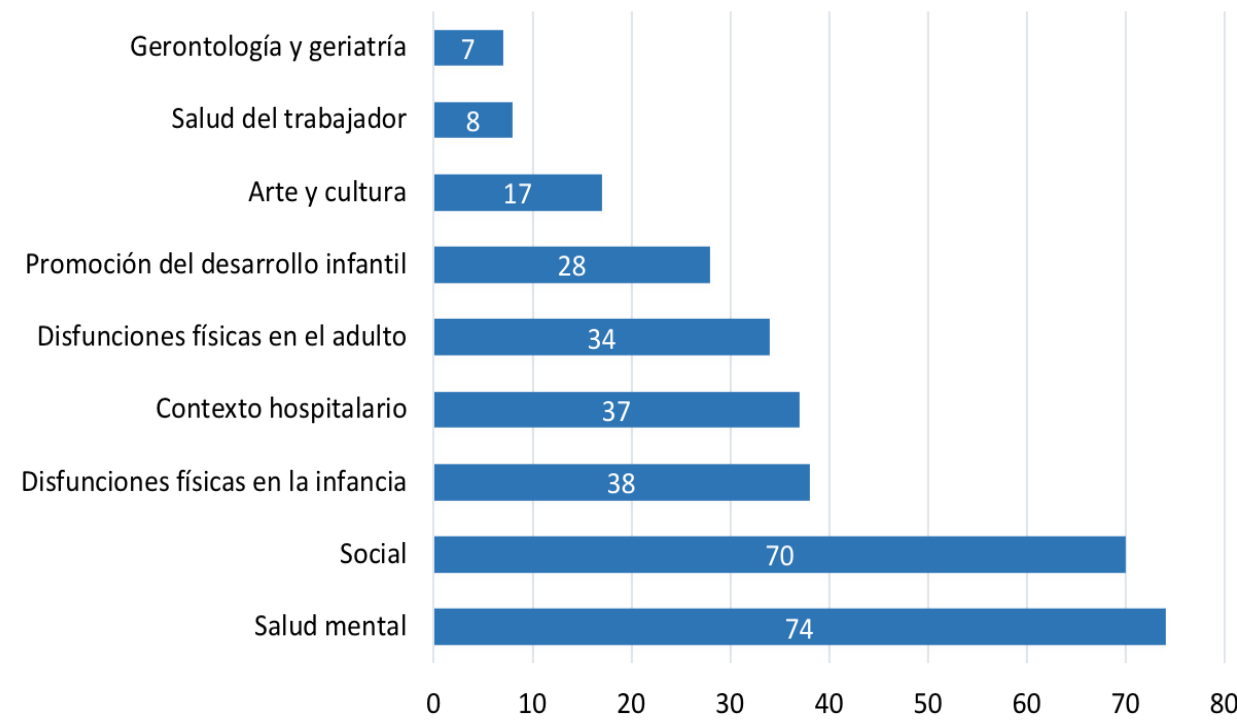

Fuente: elaboración propia.

En relación con las actividades destinadas a la comunidad y las prácticas de Terapia Ocupacional, se evidenció que se concretaron en distintos campos $\mathrm{o}$ áreas (figura 2).

En la figura 2 puede observase que los campos o áreas en los que menos actividades se han desarrollado están relacionados con que ha habido un o una única docente vinculada al Departamento de Terapia Ocupacional en la última década.

El público prioritario de las actividades es amplio y diverso, la extensión tiene gran alcance y logra involucrar a un número significativo de personas de la comunidad interna o externa a la Universidad. La suma total fue de 74.682 participaciones de personas en diferentes cursos de vida, perfiles y categoriza- ciones (figura 3), considerando que es posible que una misma persona participe de diferentes actividades. Los registros revelan 24 actividades cuyo público objetivo fueron bebés; 39 en la que fueron niños y niñas: 36 , adolescentes; 29, jóvenes; 248, personas adultas, y 14 dirigidas a personas mayores. Además, una misma actividad podía ser ofrecida para públicos distintos.

En 124 actividades se vincularon personas externas al Departamento de Terapia Ocupacional, en 55 de ellas se involucraron personas de la comunidad; se contó con 55 participantes de servicios u organizaciones externas (como asociaciones de profesionales, personas de colectivos de la comunidad, organizaciones civiles, entre otros) y con 37 docentes jubilados o voluntarios y funcionarios de la Universidad. 
Las actividades comunitarias estuvieron muy presentes, 163 ocurrieron en la propia universidad, 150 dentro y fuera, 60 solo fuera, 50 en otras ciuda$\operatorname{des}^{7}$ y una actividad se realizó por enseñanza a distancia (en línea).

Las actividades externas ocurrieron en diferentes espacios, 198 fueron realizadas en servicios públicos, entre ellos: 106 de asistencia social o de salud, como en centros de apoyo, protección y espacios culturales para la niñez y la juventud, centros de atención o servicios en salud o de asistencia social destinados a poblaciones específicas; 46 en instituciones de educación como guarderías y escuelas de diferentes niveles; 29 en hospitales, y otras 17 en universidades o facultades.

Figura 3. Perfil del público involucrado en las actividades de extensión.

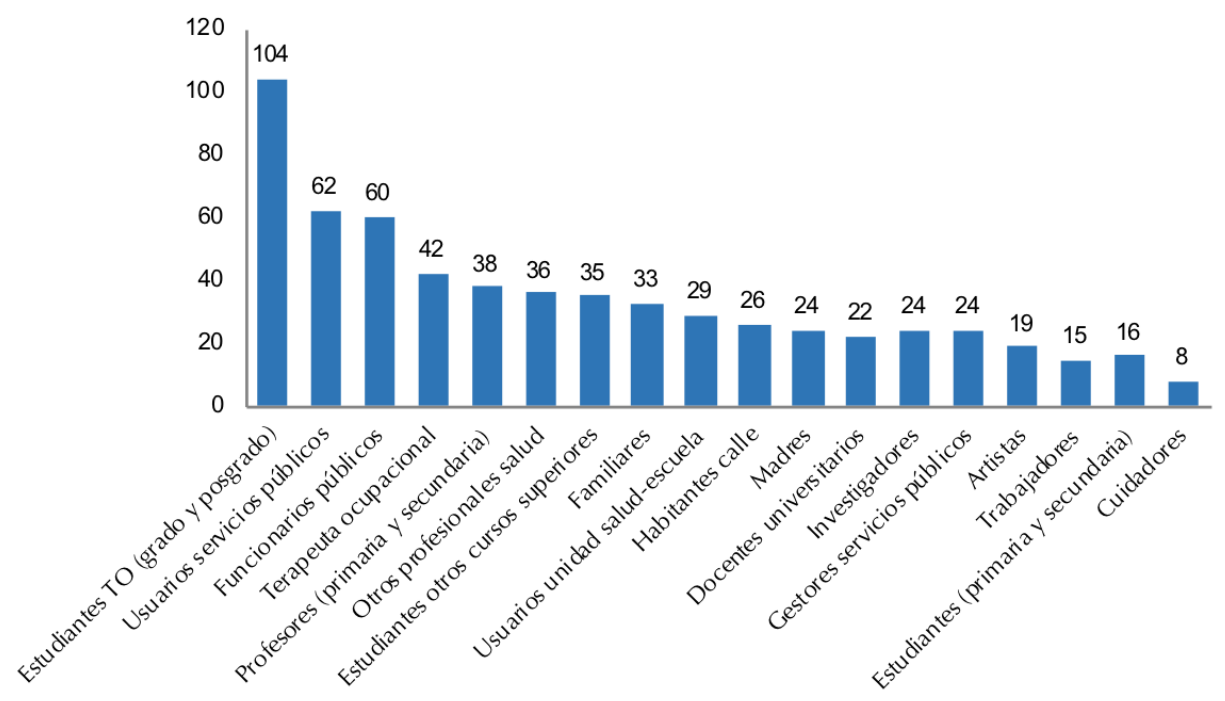

Fuente: elaboración propia.

\section{Actividades de extensión y formación académica}

Las y los estudiantes disfrutan de la oportunidad de participar de las actividades de extensión a través de las prácticas y de estudios en cualquier mo- mento de la formación de los cursos de pregrado y posgrado. De esta manera, adquieren experiencia y su formación se vuelve más rica y comprometida con la sociedad; adicionalmente, pueden obtener una beca.

\footnotetext{
${ }^{7}$ En siete actividades no se identificó el lugar en el que fueron realizadas.
} 
Cada año la Universidad abre convocatorias para la selección de actividades de extensión. Las propuestas son evaluadas, a partir de criterios preestablecidos, para definir la distribución de ayudas financieras y de becas destinadas a estudiantes de pregrado.

Fueron mapeados 1.367 estudiantes que participaron de la ejecución de las actividades de extensión, entre ellos, 818 becarios $^{8}, 236$ estudiantes de posgrado y 39 de otras instituciones de enseñanza superior.

La mayoría de las actividades de extensión (228) se realizaron únicamente con la o el docente coordinador, 190 contaron con la participación de otros docentes del mismo departamento, 31 con docentes de otros departamentos de la Universidad (figura 4) y 20 actividades fueron coordinadas por pares de otros departamentos y en ellas participaron docentes de Terapia Ocupacional. Además, hubo 54 docentes de otras instituciones de enseñanza superior que se involucraron en 13 actividades de extensión.

Otros 31 sectores de la Universidad también participaron en las actividades, se destaca que la Unidad de Salud Escolar, que es el servicio ambulatorio localizado y gestado por la Universidad, fue citada en 49 proyectos.

Además del apoyo de los sectores al interior de la comunidad universitaria, se identificaron algunos externos registrados: 131 actividades con organismos públicos especificados y otros 12 no especificados; 17 órganos privados con especificación y uno sin ella; 14 alianzas con el tercer sector ${ }^{9}$ y organizaciones no gubernamentales; 5 con movimientos sociales, y 20 con registros diversos, como empresas, programas, asociaciones, proyectos y sectores públicos. 212 actividades no reportaban alianzas externas.

\section{Discusión}

Los resultados demuestran una gran cantidad de actividades de extensión realizadas por el Departamento de Terapia Ocupacional de la Universidad Federal de São Carlos, abarcando diversas áreas, objetivos, públicos, espacios y asociaciones.

Ante las actividades de extensión analizadas en los últimos 20 años, se observa una amplia integración de la profesión con otras áreas del conocimiento y con la formación práctica propia. Así, se observan asociaciones con otros departamentos de la Universidad, con órganos y sectores públicos y de la sociedad civil. Con ello se cumplió la principal meta de las actividades de extensión, que es la relación con la comunidad, con los más diversos perfiles de poblaciones, ofreciendo inversiones, cambios y acciones sociales.

De otro lado, se contó con la participación fundamental de estudiantes que contribuyeron con las propuestas

\footnotetext{
${ }^{8}$ En 102 registros no constaba esa información.

${ }^{9}$ Tercer sector es un término sociológico utilizado para definir iniciativas privadas sin ánimo de lucro que prestan servicios de carácter público, conocidas como asociaciones, fundaciones, instituciones autónomas y organizaciones no gubernamentales (Albuquerque, 2006).
} 
Figura 4. Departamentos involucrados en las actividades de extensión coordinadas por docentes del Departamento de Terapia Ocupacional.

- Psicología

- Enfemería

- Ingeniería de Producción Medicina

Artes y Comunicación, Fisioterapia

- Filosofía y Metodología de las Ciencias

Ingeniería Civil, Educación Fís ica, Computación, Gerontología, Ciencias Fisiológicas, Ciencias Ambientales, Pedagogía, Gerontología, Unidad de Atención Infantil

Fuente: elaboración propia.

$y$, al mismo tiempo, favorecieron su formación profesional, en acuerdo con Fresán (2004).

De esta manera, al mismo tiempo que las universidades asumen la extensión como la articulación de las distintas tareas universitarias y la vinculación entre estas y la sociedad, podrían explotar posibilidades casi infinitas de acción para ampliar sus ámbitos de influencia y contribuir a la educación, no solo de jóvenes, sino de individuos de todas las edades, formando seres humanos capaces de descubrir y reafirmar día a día su saber, de mirar críticamente la realidad y de participar en los procesos de transformación social (Fresán, 2004).

En relación con lo anterior, quienes han coordinado las actividades de extensión han generado una serie de publicaciones en las cuales relatan sus experiencias a través de la divulgación científica en el área. Algunas de ellas presentan las contribuciones de las actividades de extensión a la formación en Terapia ocupacional. Por ejemplo, Della Barba et al. (2017) evaluaron que el proceso de enseñanza-aprendizaje 
ocurrido en un proyecto de extensión contribuyó con la interacción entre estudiantes, usuarios y equipos de los servicios de salud involucrados. Desde el punto de vista de la formación de las y los alumnos, el proceso facilitó la comprensión sobre el Sistema Nacional de la Salud y potenció las discusiones sobre la actuación de la Terapia Ocupacional en la comunidad.

Entre tanto, Lopes et al. (2011) discuten que el desarrollo de la extensión posibilitó la creación de vínculos significativos entre el estudiantado y la población, desdoblándose en acompañamientos individuales, en la ampliación de sus redes de soporte social y en la producción de espacios de convivencia que favorecieran el respeto, el diálogo y la comprensión de una nueva perspectiva sobre cómo se dan las relaciones con el otro.

Por otra parte, los datos recolectados demuestran la diversidad de los proyectos de extensión, tal como lo establece el principio de indisociabilidad entre enseñanza, investigación y extensión, lo que contribuye al reconocimiento de la Terapia Ocupacional en sus prácticas. Los hallazgos también señalan que el equipo docente ha invertido en la divulgación de tales prácticas y de sus resultados, favoreciendo el reconocimiento de la profesión en el escenario académico y alentando la realización de nuevas propuestas.

Cabral (2012) señala los retos y las perspectivas de la extensión y la afirma como práctica social de la universidad que tiene sentido si se inserta en la propuesta pedagógica, asociada a la formación, o se convierte en función de las políticas públicas, no de la educación y la ciencia. Así, la extensión tiene la necesidad de transformar los procesos de conocimiento, asociada con la enseñanza y la investigación, en la búsqueda de alternativas para su democratización como bien público.

Sin embargo, no siempre se ofrecen las condiciones ideales para la ejecución de la extensión, sobre todo para aquellas actividades que no serán financiadas desde la lógica del mercado, pues no producen una riqueza convertida en moneda y ganancia, no obstante, redundan en crecimiento humano y social. Estas actividades tienen dificultades para su financiación y sostenibilidad, considerando además que el financiamiento ha sido insuficiente frente a la demanda de la universidad. Se observa también que las universidades, en su autonomía, tienen sus propios métodos y estrategias para el financiamiento de estas actividades.

Serna (2007) propone cuatro modelos de extensión universitaria en las universidades en América Latina, una vez que los fundamentos, actividades y finalidades de la educación han tenido grandes cambios: i) altruista, con influencia positivista, estructura las prácticas desinteresadas y humanitarias de los universitarios en favor de poblaciones "pobres" o "ignorantes"; ii) divulgativo, la supuesta preocupación de las universidades por elevar la cultura de la población, y por tanto su bienestar, difícilmente se revela como respuesta a las auténticas necesidades y anhelos de los sectores mayoritarios de la sociedad, es decir, los pobres o en situación de vulnerabilidad; iii) concientizador, modelo inspirado por el pensamiento de izquierda latinoamericano y por Freire, que pondera compartir los bienes, incluidos los educativos y 
culturales, en forma dialógica y liberadora, creando conciencia entre quienes participan en ese acto educativo, y iv) vinculatorio empresarial, desde la mitad de los años 80 la relación con las empresas empezó a fomentarse y a ser redituable para las universidades, en este modelo las necesidades sociales son identificadas como análogas a las de las empresas y, en consecuencia, las y los universitarios deberán ser educados para atender a estas necesidades.

Para Ortiz y Morales (2011), las actividades de extensión en las universidades latinoamericanas se dan en tres enfoques: empresarial, social y cultural; sin embargo, el modelo económico dominante ha hecho que estas instituciones respondan a las necesidades de su entorno local, regional y nacional, pero es evidente una tendencia hacia el desarrollo de la función de extensión desde el enfoque empresarial, como un requisito del mercado y del sector productivo. Estas autoras buscan demostrar que el privilegio de dicho enfoque en los últimos años, impuesto por las dinámicas del mercado y muy destacado en las universidades anglosajonas, no considera que Latinoamérica tiene condiciones particulares que requieren del liderazgo de las universidades en la solución de problemas sociales y la conservación de su acervo cultural.

Chauí (2003) también hace críticas severas al modelo empresarial, dictado por el sistema neoliberal, que ha transformado la enseñanza superior y la universidad pública, pues defiende la destinación del fondo público a los derechos sociales, garantizada por la democratización del Estado y, con ella, de la propia universidad.
Así, el real papel de la extensión universitaria, sumado a las características actuales de financiamiento de la universidad pública, se convierten en el núcleo de la cuestión sobre lo que será incentivado y promovido como tal. Para la Terapia Ocupacional esta es una cuestión importante, ya que la mayoría de sus propuestas están más relacionadas con el intercambio de saberes y prácticas y con la formación, que con actividades de venta de servicios o equipamientos, propios de un enfoque empresarial.

En ese sentido, es importante reafirmar que la perspectiva de la educación es humanizar sujetos a partir de la acción consciente, para que puedan transformar el mundo; según Freire, "la extensión de sus conocimientos y de sus técnicas posibilita a los hombres que puedan transformar mejor el mundo en el que están" (1985, p. 11).

Además, la superación del papel eminentemente técnico significó para las y los terapeutas ocupacionales brasileños la conquista ética y política de profesionales quienes, en su compromiso con la redemocratización del país, lucharon por los derechos de las poblaciones vulnerables, estigmatizadas y marginadas. Estas personas acudieron a las calles y alcanzaron nuevos espacios más allá de las instituciones, con muchas posibilidades de actuación (Galheigo, 2016); realidad muy presente para la Terapia Ocupacional en Latinoamérica y sus prácticas comunitarias.

En los últimos 30 años se revisaron concepciones, como la de salud, haciendo más compleja la visión del ser humano y de sus condiciones de bienestar. Estas transformaciones fueron in- 
corporadas en el desarrollo de políticas públicas y en las prácticas profesionales, situando a individuos y comunidades como sujetos activos y protagonistas de las acciones (Apablaza, 2015).

\section{Conclusiones}

El presente estudio dejó en claro que la extensión universitaria ha posibilitado a las y los docentes de la carrera de Terapia Ocupacional de la Universidad Federal de São Carlos desarrollar acciones y proyectos comunitarios insertados en diferentes servicios públicos y sectores, incluso donde no hay terapeutas ocupacionales.

En este sentido, las actividades de extensión han promovido el acceso, la permanencia y la cualificación de los servicios públicos, ampliado las acciones ofrecidas por estos espacios. También han ayudado a la formación continua de profesionales, desarrollando asistencia a la comunidad a través de la integración entre enseñanza, investigación y extensión universitaria. Además, han contribuido a la divulgación y a la ampliación de las posibilidades de actuación de la Terapia Ocupacional con las comunidades.

Cabe señalar que el sector público ha experimentado numerosas dificultades para mantener los servicios básicos de asistencia social, salud y educación para la población, al tiempo que los sectores del deporte, la cultura y el ocio siguen siendo un privilegio reservado a unos pocos, aun cuando sean derechos garantizados en la Constitución Nacional brasileña. Con esto, se considera que el trabajo docente debe estar com- prometido ética y políticamente con la producción y la transferencia de conocimiento, desde la relación dialógica con la comunidad y la integración de las actividades de enseñanza, investigación y extensión, de manera crítica y como parte de su función social, consciente de su papel académico, político, ético y profesional.

Es necesario contar con profesionales comprometidos con la construcción y la expansión constante de las políticas públicas y de los derechos sociales y humanos que promuevan una mayor democratización de los bienes públicos, la participación social y la autonomía de sujetos y colectivos. Para las y los terapeutas ocupacionales este es un presupuesto profesional y un compromiso ético y político.

Ante estas consideraciones, son contundentes las contribuciones de la extensión para la cualificación de la formación en Terapia Ocupacional y como política de la universidad pública. Sobre todo en los espacios de la comunidad, donde la ocupación, las actividades humanas y la producción de la vida ocurren, la extensión ofrece innumerables posibilidades de acción para la profesión, en las que las poblaciones involucradas se benefician de los procesos de enseñanza-aprendizaje.

Es importante, entonces, que la ciudadanía involucrada en actividades de extensión considere que las políticas públicas educativas deben permitir, promover y valorar que la universidad cumpla su rol y su función social.

Futuras investigaciones pueden ayudar a comprender las contribuciones de diferentes actividades comunitarias a la 
formación de docentes y estudiantes, dentro del alcance de la extensión universitaria y la vinculación con el medio. Algunas preguntas que podrían orientar esos estudios son: ¿qué preguntas no se han podido resolver con los saberes de la Terapia Ocupacional en las comunidades?, ¿cuáles saberes, presentes en las comunidades, necesitan ser incorporados en procesos dialógicos con la Terapia Ocupacional?, ¿qué soluciones ocupacionales ante la precarización laboral se gestionan en las comunidades?, ¿cómo y qué practicas comunitarias favorecen la participación social, la ciudadanía y la democracia?

Finalmente, los datos recabados a través de este estudio proveen información útil para posteriores evaluaciones, investigaciones, comparaciones y proyecciones. También, permiten recuperar la memoria y contribuyen a la preservación del patrimonio histórico y cultural de la Universidad Federal de São Carlos, aumentando la visibilidad de las acciones de extensión de Terapia Ocupacional y de la institución.

\section{Financiación}

Artículo resultante de un proyecto de iniciación científica con fondos de la Fundación de Apoyo a la Investigación del Estado de São Paulo (FAPESP).

\section{Referencias}

Albuquerque, A. C. C. (2006). Terceiro setor: história e gestão de organizações. Summus.

Apablaza, M. (2015). El desafío de formar: una reflexión desde la práctica como formadora de terapeutas ocupacionales. Revista Chilena de Terapia Ocupacional, 15(2), 1-17. https://revistas.uchile.cl/index.php/ RTO/article/view/38156

Cabral, N.G. (2012). Saberes em extensão universitária: contradições, tensões, desafios e desassossegos [Tesis de doctorado, Universidade Federal do Rio Grande do Sul]. Repositório Digital UFRGS. https://lume.ufrgs.br/handle/10183/49409

Cardinalli, I. (2017). Conhecimentos da Terapia Ocupacional no Brasil: um estudo sobre trajetórias e produções [Tesis de maestría, Universidad Federal de São Carlos). Repositorio Institucional UFSCar. https://repositorio.ufscar.br/handle/ufscar/8496

Chauí, M. (2003). A universidade pública sob nova perspectiva. Revista Brasileira de Educação, (24), 5-15. http://www.scielo. br/pdf/rbedu/n24/n24a02.pdf

Coordenação do curso de bacharelado em Terapia Ocupacional (2011). Projeto pedagógico do curso de graduação em terapia ocupacional. Universidade Federal de São Carlos. http://www.prograd.ufscar. br/projetoped/projeto_TO.pdf

Conselho de Extensão da Universidade Federal de São Carlos (2016, 17 de marzo). Resolução do CoEx $n^{\circ}$ 03/2016, de 17 de março de 2016. Aprova o Regimento Geral da Extensão da Universidade Federal de São Carlos. http://www.proex.ufscar. br/arquivos/normas-regras-e-outros/reso- 
lucao-coex-03-17-03-2016-regimento-geral-da-extensao.pdf

Della Barba, P.C.A., Barros, V.M., Luiz, É.A.M., Farias, A.Z., Aniceto, B. y Miyamoto, E.E. (2017). Terapia Ocupacional em um processo de capacitação sobre vigilância do desenvolvimento infantil na atenção básica em saúde. Cadernos de Terapia Ocupacional da UFSCar, 25(1), 223-233. https://doi.org/10.4322/0104-4931.ctoRE0747

Farias, J. P (2015). Extensão universitária como mecanismo de desenvolvimento educacional e social no Brasil. Fragmentos de Cultura, 25(1), 75-82. http://dx.doi. org/10.18224/frag.v25i1.4158

Fausto, B. (2009). História do Brasil (13ª ed.). Editora da Universidade de São Paulo.

Fleuri, R.M. (1989). Extensão universitária em educação popular. Educação e Filosofia, 3(5/6), 59-73.

Fórum de Pró-Reitores de Extensão das Universidades Públicas Brasileiras [Forproex] (2006). Indissociabilidade ensino-pesquisa-extensão e a flexibilização curricular: uma visão da extensão. MEC/SESu.

Freire, P. (1985). Extensão ou comunicação? ( ${ }^{a}$ ed.). Paz e Terra.

Freire, P. (1987). Pedagogia do oprimido (17 ed.). Paz e Terra.

Fresán, M. (2004). La extensión universitaria y la universidad pública. REencuentro. Análisis de Problemas Universitarios, (39), 47-54. https://www.redalyc.org/articulo.oa?id=34003906

Galheigo, S.M. (2016). Terapia ocupacional social: uma síntese histórica acerca da constituição de um campo de saber e de prática. En: R.E. Lopes y A.P. Malfitano (org). Terapia Ocupacional Social: desenhos teóricos e contornos práticos (pp.49-68). EdUFSCar.

Goodwin, J.C. (1995). Research in psychology: Methods and design. John Wiley.

Harvey, D. (2014). O neoliberalismo: história e implicações. Loyola.

Junior, A.L.S. (2013). A extensão universitária e os entre-laços dos saberes [Tesis de doctorado, Universidade Federal da Bahia]. Repositorio UFBA. https://repositorio.ufba.br/ri/handle/ri/17554

Llorens, L. (1992). Planeación y extensión universitaria. Revista de la Educación Superior, 20(81), 3-15. http://publicaciones.anuies. mx/pdfs/revista/Revista81 S1A4ES.pdf

Lopes, R.E., Borba, P.L.O., Trajber, N.K.A, Silva, C.R. y Cuel, B.T. (2011). Oficinas de atividades com jovens da escola pública: tecnologias sociais entre educação e terapia ocupacional. Comunicação Saúde Educação, 15(36), 277-88. http://dx.doi.org/10.1590/S1414$\underline{32832011000100021}$

Minto, L W (2011). A educação da "miséria": particularidade capitalista e educação superior no Brasil [Tesis de doctorado, Universidade Estadual de Campinas]. Repositorio Unicamp. http:// repositorio.unicamp.br/jspui/bitstream/ REPOSIP/251175/1/Minto LaloWatanabe D.pdf

Nogueira, M.D.P. (2001). Extensão universitária no Brasil: uma revisão conceitual. En: D.S. Faria (org.), Construção conceitual da extensão universitária na América Latina (pp.57-72). UNB. 
Ortiz, M.C. y Morales, M.E. (2011). La extensión universitaria en América Latina: concepciones y tendencias. Educación y Educadores, 14(2), 349-366. http://educacionyeducadores.unisabana.edu.co/index.php/eye/article/view/1928/2515

Paula, J.A. (2013). A extensão universitária: história, conceito e propostas. Interfaces, 1(1), 05-23. https://www.ufmg.br/proex/ revistainterfaces/index.php/IREXT/article/ view/5/pdf

Pedrazzi, V. y Yamanoto, M.M. (2013). A necessidade de ações articuladas na cultura e extensão. Revista Cultura e Extensão USP, 10(1), 43-50. http://dx.doi.org/10.11606/ issn.2316-9060.v10i0p43-50

Presidência da República (1988). Constituição da República Federativa do Brasil de 1988. http://www.planalto.gov.br/ccivil 03/constituicao/constituicaocompilado.htm

Serna, G.A. (2007). Misión social y modelos de extensión universitaria: del entusiasmo al desdén. Revista Iberoamericana de Educación, 43(3), 2-7. https://dialnet.unirioja. es/servlet/articulo?codigo $=2316425$

Silva, A.F.L., Ribeiro, C.D.M. y Silva Junior, A.G. (2013). Pensando extensão universitária como campo de formação em saúde: uma experiência na Universidade Federal Fluminense, Brasil. Comunicação Saúde Educação, 17(45), 371-84. http://dx.doi. org/10.1590/S1414-32832013000200010

Soares, L.B.T. (1991). Terapia Ocupacional: lógica do capital ou do trabalho? Hucitec.

Sousa, B.S. (2007). La universidad en el siglo XXI para una reforma democrática y emancipatoria de la universidad. $\mathrm{Cl}$ DES-UMSA, ASDI y Plural editores.
Tünnermann, C (2008). Noventa años de la Reforma Universitaria de Córdoba: 1918-2008. CLACSO. 